\title{
Logging Kernel Events on Clusters
}

\author{
Jürgen Reuter and Walter F. Tichy \\ Institut für Programmstrukturen und Datenorganisation \\ Fakultät für Informatik \\ Universität Karlsruhe, 76128 Karlsruhe, Germany \\ $\{$ reuter,tichy\}@ipd.uka.de \\ http://www.ipd.uka.de/ $\{$ reuter, tichy $\} /$
}

\begin{abstract}
We present tools for recording and analysing kernel events on Linux clusters. The tools provide cluster-wide event logging at system clock accuracy. We demonstrate the usefulness of our tools by verifying an implementation of a simple remote scheduling feature and present interesting results regarding schedule responsiveness. With our GUI-based Java application, data recorded on multiple hosts is integrated for visualization. These tools can be used for analysis of cluster schedulers such as gang schedulers, cooperative cacheing, network RAM drivers, or parallel file systems.
\end{abstract}

\section{Introduction}

Tracking and analysing events on computer clusters is essential for understanding and improving crucial aspects of cluster operating systems, such as gang scheduling, synchronization, parallel file access, managing network RAM, or cooperative cacheing. We provide a set of tools that collect and display kernel events on clusters of Linux computers. The events are logged by kernel modifications on each node individually and integrated in a cluster-wide view for analysis. Our current implementation captures scheduling events and is intended to help understand gang scheduling strategies. Other events can also be captured, such as block lookup and eviction requests in cacheing policies of network RAM systems on globally coordinated cluster caches. Similarly, collecting and analysing information regarding parallel access to files may help choose proper policies for I/O scheduling, cacheing and prefetching. Our event recording approach achieves system clock resolution.

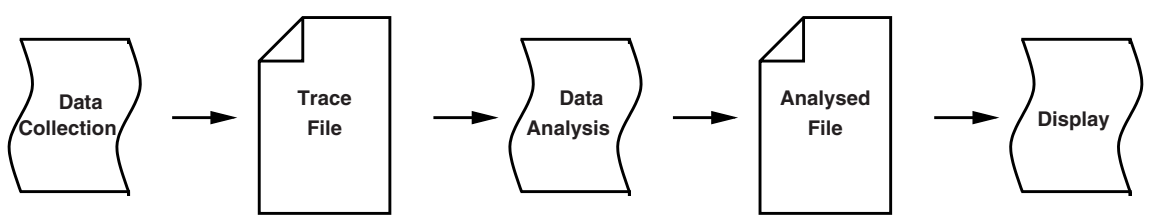

Fig. 1. General Performance Analysis Approach 
Traditionally, post-mortem performance analysis follows a pattern as shown in Figure 1. The first step is to collect data, which can be done in manifold ways. For example, user applications can be instrumented, i.e. additional code may be inserted at selected locations in the application such that when execution comes across such location, timestamp and program status information is recorded and appended to a logfile. This way, the logfile subsumes a trace of selectively recorded events in the course of program execution. Rather than inserting code for instrumentation into a user application, one can also insert such code at relevant locations of underlying libraries. For example, by instrumenting a communication library such as PVM[10] or MPI[5], one can track the communication pattern of a user application that uses the library, but may not be able to find bottlenecks in the communication itself. Our approach is to instrument selected locations of the OS kernel, gaining a better understanding of specific performance issues in the cooperation between the kernel and user applications.

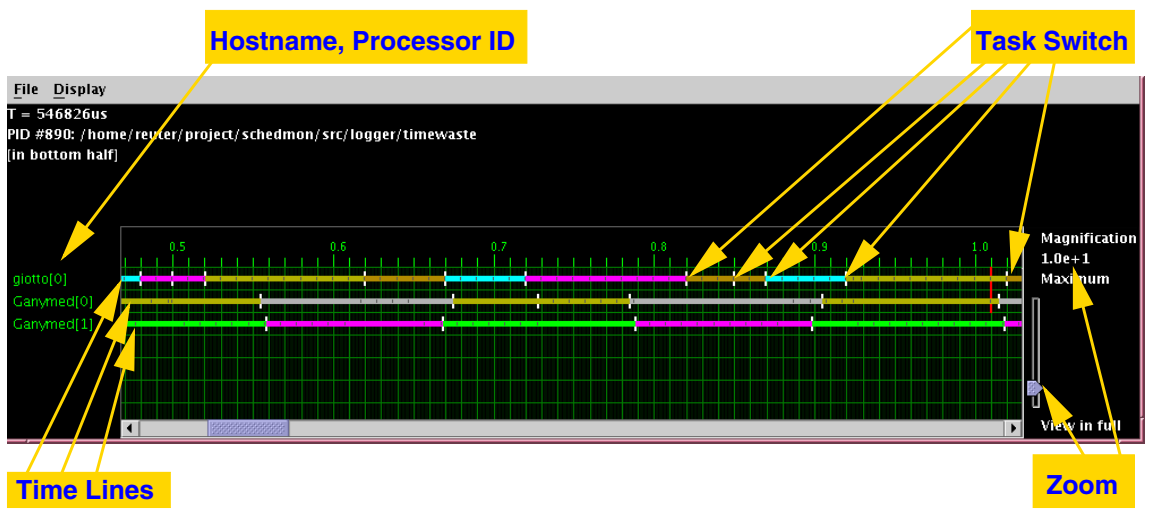

Fig. 2. Data Visualization Tool

The second step in the course of post-mortem performance analysis, the data analysis, consists in filtering events, as well as computing some statistics. The third step is to display the results of the analysis. We integrated these final two steps into a single Java application with a graphical user interface. Data display is based on showing events on per-processor time lines, as shown in Figure 2.

The remainder of this paper is organized as follows: Section 2 gives an overview of the kernel modifications and a companian user level application to record event data. In Section 3, we present the visualization tool for analysing and displaying the data. As an example of usage, in Section 4 we show how we evaluated the performance of a remote scheduling feature with our tools. Section 5 discusses some limitations of our current design and presents possible solutions that we envision for the future. In Section 6, we compare our work with related approaches. Section 7 summarizes the results of this work. 


\section{Data Collection}

The data collection process is realized by recording local time and type of kernel events through instrumentation of the kernel. Our work focuses on instrumenting selected locations in the kernel code rather than instrumenting user level applications. With this approach, we can efficiently log data that is gathered in the kernel without suffering from the additional overhead of switching between kernel and user mode. The possibly most important type of event and relevant motivation for this work is the point of time when the kernel, while executing the schedule() function, decides to switch from one task to another one. Similarly, we may want for example to observe when an ICMP message arrives from the network, or how much time the kernel spends in a particular servicing routine, e.g. by recording when the kernel enters and leaves bottom halves ${ }^{1}$ as part of the do_softirq() kernel function.

For this purpose, we provide a framework consisting of kernel code (based on modifications of a SuSE 8.0 Linux 2.4.18 kernel) that allows for recording such events, and a user level application that is responsible for saving the data to disk. This framework follows the producer-consumer paradigm, but is relaxed in the sense that the producer can not block, but will rather discard data when its buffer overflows.

From the perspective of the kernel code to be instrumented, we simply provide a new kernel function evtlog_put (struct evtlog_entry_t *entry, int/*bool*/ fill_in_time), where struct evtlog_entry_t entry consists of an event id, a timestamp and 8 bytes of event specific data. Depending on the boolean flag, the timestamp is either provided by the caller or automatically filled in by calling do_gettimeofday(). Upon calling evtlog_put(), a copy of the struct is saved in a kernel memory buffer. The function is designed to return quickly, such that the overhead of recording does not affect the collected data too much. Therefore, when the buffer is about to overflow, it does not try to save the data to disk by itself. Instead, the kernel function wakes up a dedicated user level process that is responsible for fetching the data from the kernel and saving it to disk.

Figure 3 illustrates the interactions between the kernel logger code and the user level logging process. It shows an SMP machine with two processors. The left side represents the kernel logger code, the right side the dedicated user level logger process. The user level logging process sleeps by invoking pause (), waiting to be notified by the kernel upon arrival of log data. For each processor, the kernel logger code provides memory for two buffers. Upon invocation, the kernel logging function writes any event data into the first buffer of the currently executing processor. When this buffer is full, it swaps the two buffers by exchanging pointer references, then makes the user level logging process runnable by calling the kernel function wake_up_process (), and finally returns to the caller. Eventually,

\footnotetext{
${ }^{1}$ Under Linux, a bottom half designates the non-critical part of an interrupt handler that is not executed immediately when the interrupt occurs, but is delayed until all time critical code has been executed.
} 


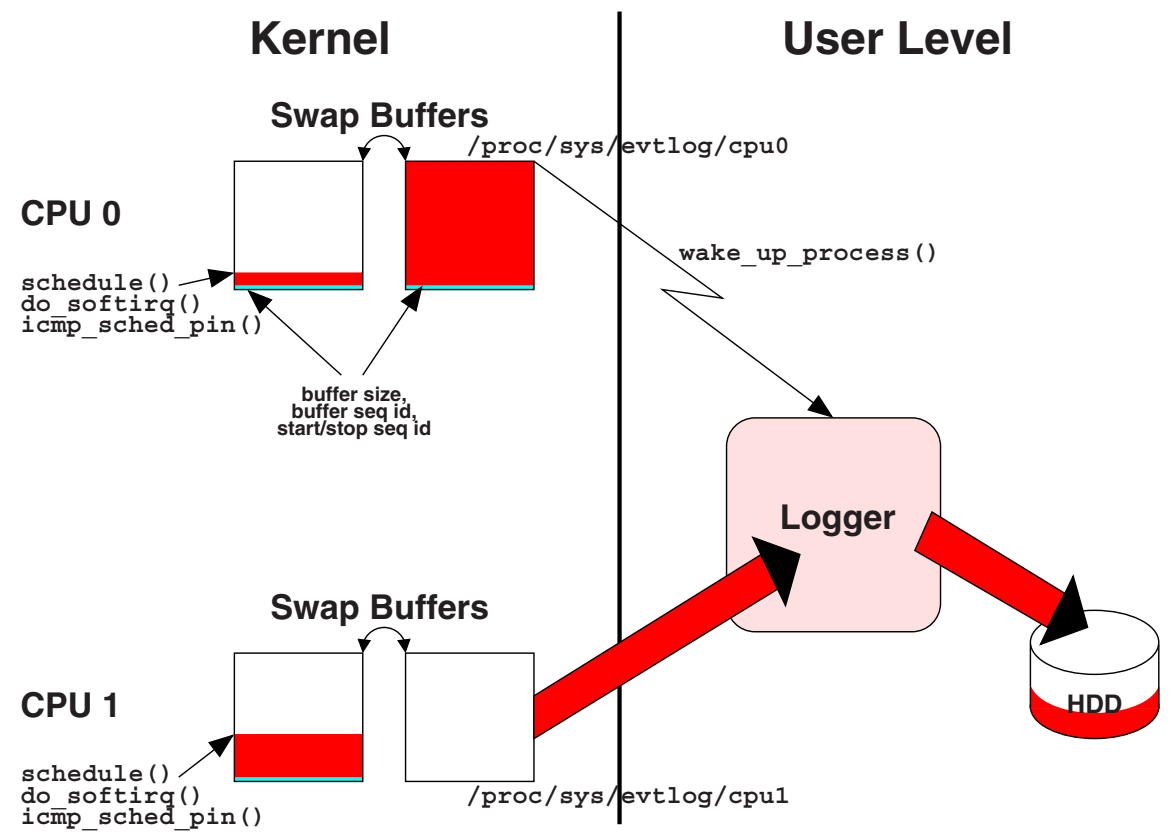

Fig. 3. Interactions between kernel logging code and user level logging process

the kernel scheduler will switch to the user level logging process, since it is now runnable. The user level logging process then looks for any data available for any processor from the kernel logger, fetches it, writes it to disk, and puts itself back to sleep by invoking pause() once again. In the current implementation, the data is transferred from kernel to user space by copying it in the course of reading from a dedicated file in the $/$ proc $^{2}$ filesystem, such that user level applications can initiate read or write access filesystem. When reading from this file, a boolean flag is reset in the kernel, telling it that the buffers may be swapped once again. A future implementation may avoid the extra copy through the /proc filesystem by the kernel directly writing the log data into user space memory that has been allocated by the user level logging process.

The user level logging process has to announce itself to the kernel such that the kernel logger code knows which process to wake up upon a full buffer. This is done during startup of the user level logging process through, once more, a special file entry in the /proc file system: the user level logging process writes its own process id to that file.

Data may be discarded if the kernel records data faster than the user level logging process is capable of saving, as the process is scheduled a limited amount

${ }^{2}$ The Linux kernel provides pseudo file entries in the /proc filesystem, such that user level applications may exchange data with the kernel by writing to or reading from a file in this filesystem. 
of time only. To be able to detect such data loss, each buffer contains a perprocessor sequence number which is increased whenever the kernel starts writing a new buffer. Checking the sequence numbers for completeness is left to the visualization tool, since the logging itself is designed to produce little overhead.

Kernel logging can be dynamically turned on and off. This mechanism is also done via the /proc file system by writing to special files. Whenever kernel logging is restarted, the user level logging process creates a new log file on disk and writes subsequent buffers to it. For that purpose, in addition to the buffer sequence number, there is also a start/stop sequence number that is incremented each time kernel logging is turned on, such that the user level logging process can determine the correct log file. This is necessary, since on an SMP machine, the buffer for one processor may still contain data from an earlier logging phase as compared to another processor.

Furthermore, since upon stopping kernel logging all buffers are flushed, a third special entry is needed that represents the actual size of the current buffer. Choosing a null-terminated array for the buffer implementation is not reasonable, since this would require spending time for scanning through the whole buffer to determine its size when writing it do disk.

With respect to keeping the logging overhead small, the user level logging process just fetches and stores the data in whatever order it retrieves it from the kernel. It only cares for evaluating the start/stop sequence number for writing the data to the correct file.

Since the kernel logger uses per-processor buffers, on an SMP machine, kernel logging can be performed concurrently among all processors without requiring a time-wasting SMP kernel lock: the kernel logging function accesses always the buffers of the currently executing processor, thus scaling fine even on fat SMP nodes. Calling the kernel logging function from multiple locations including bottom half code is possible, since the kernel itself is not preemptive, such that calls to the kernel logging function are executed sucessively without overlapping in time. However, with the current implementation, the kernel logging function should not be called from within a hardware interrupt handler, since the handler might have interrupted a pending call of the kernel logging function. Overlapping calls to the kernel logging function effectively have the same effect as unsynchronized concurrent access to the log buffers, thereby potentially damaging its consistency. Preventing such a scenario requires additional provision such as queuing logging requests. This may be added in a future version.

Our kernel modifications include a call to the kernel logging function whenever the Linux scheduler decides to switch to another process on any processor. This way, the processing time of the user level logging process itself is logged and can be used for determining the logging overhead caused by the user level logging process. This includes the time needed for copying the log data from kernel space to user space, since the read access on the / proc file system is executed in the context of the user level logging process.

The overhead in the kernel itself can not be tracked this way. But since the kernel logging function mostly consists of determining the timestamp and writing 
a log entry into kernel memory, it can be well estimated by assuming a constant cost of time per log entry for a particular machine. Our experiences suggest that determining the timestamp is a bottleneck, but that obviously depends on the particular hardware.

Since logging is performed locally on each node (assuming that the data is written to a local file system), the system scales fine with an increasing number of cluster nodes. On fat SMP nodes, writing the data to disk may become a bottleneck (though Linux supports only up to 32 processors). Still, if the logging overhead is roughly the same for all nodes, scheduling all logging processes simultaneously as a gang can be used to minimize the effect of the logging overhead onto any other processes of the system.

\section{Data Analysis and Display}

The user level logging process creates log data files by retrieving data from the kernel via the /proc file system and writing it to disk without further processing. It is the task of separate tools to reorder, filter, evaluate and display the data. For this purpose, we provide a standalone Java application with a graphical user interface. Since it is written in a platform independent manner, it runs on several platforms. This is handy, when, for example, the log data is collected on a Linux cluster, but the perfomance analysis will be done on a separate working station that potentially runs under a different OS.

Figure 2 shows a screenshot that depicts typical use of the Java tool. For each processor, grouped on a per host basis, a graphical display panel shows a horizontal time line with the currently running processes displayed in different colors. Some colors are dedicated (black = idle process, red = logger process); all other process are currently mapped to a fixed set of colors in a round-robin way, but support for user-defined color mappings may be added in a future version. Events are marked as vertical ticks across the time line, using different length and color.

The panel's view can be scrolled by either using the scrollbar below the panel or by dragging and dropping its content with the mouse. The latter is handy especially when zooming to high resolution makes the effect of scrollbar movements too coarse. The zoom of the display can be easily controlled by the logarithmicscaled slider at the right side and allows for zooming from full view to microsecond resolution. Care has been taken to ensure fast and smooth scrolling and zooming by proper design of the data structures for internal log data representation and by cacheing pointers to the data of the currently displayed location. Display of particular event types (schedule(), schedule_task(), bottom half enter/leave, ICMP schedule requests (see Section 4) and other events) can be turned on and off.

When pointing the mouse cursor on a processor's time line, the ID of the current process (and its name, if available) on that processor is textually displayed at the left side above the display panel. When positioning the mouse cursor on an event tick, additional information about the event such as the event type and its 
exact time in microsecond resolution are textually shown. Processor utilization, idle time and other general statistics are shown at program startup.

Log data from multiple hosts can be gathered via NFS: Each host writes its log file to a local disk, in order to minimize the logging overhead, and exports the directory with this file to the workstation on which the visualization tool is run. The tool may then read all log data from all hosts and display it in a single window. It is capable of displaying a large number of processor time lines, practically limited only by the amount of main memory. If memory is limited, our tool can be run with data of only a selected number of nodes that are of interest for the analysis. Filters for data reduction or extraction and combination of data from multiple processor time lines may be essential for a better understanding of huge amount of data. We are planning to implement such filters and proper visualization in a future version of the tool.

\section{Preliminary Experience}

Originally, our work was motivated by observing and controlling cluster-wide scheduling. For this purpose, we implemented a remote scheduling request feature that, for example, may be used for gang scheduling sets of related tasks of a distributed parallel application in a cluster environment. In our implementation, a host sends a special ICMP[12] message to one or several hosts to select a process group for immediate scheduling. We put additional logic into the ICMP network bottom half code of the Linux kernel such that upon reception of this special ICMP message the priority of the affected processes is set to the maximal possible value. When all bottom halves have been serviced, the Linux kernel reruns the scheduler, such that the time between receiving the ICMP message and scheduling the proper process is mostly determined by the time for processing all pending bottom halves, provided that the chance for further interrupts to intercept is negligible. On an SMP machine, the incoming ICMP message is processed by a single processor that initiates a reschedule on every other processor via a call to the kernel function smp_send_reschedule().

We used our tools to examine how much time is spent from the incoming ICMP message until scheduling the requested processes on all processors. The analysis was performed on a Dual 500MHz Pentium III (Katmai) SMP machine. We naively expected that the processor that handles the incoming ICMP message would perform the requested schedule first, since the smp_send_reschedule() would take some time before affecting all other processors.

Figure 4 shows a screenshot of our visualization tool that depicts a region of time between a particular receipt of an ICMP message and the resulting task switch. Surprisingly, the processor that handles the incoming ICMP message is often the last one that switches to the requested process. The reason may be that this processor is still in the midst of executing the network bottom half code, while the other processor on our two processor machine receives the reschedule interprocessor signal and almost immediately switches to the requested process. In any case, we could show that, when no further time is spent in servicing 


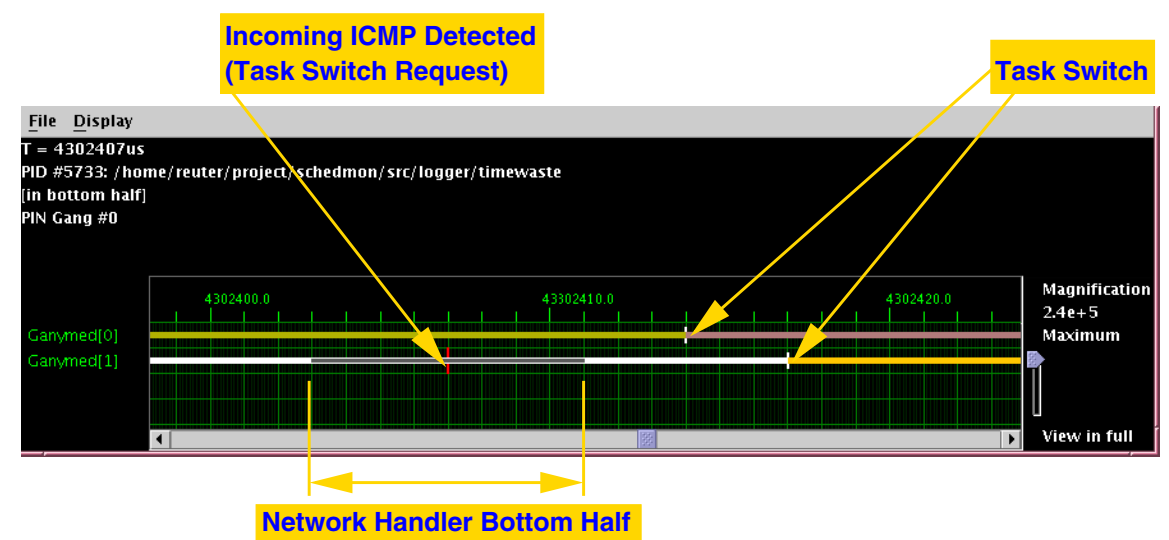

Fig. 4. Incoming ICMP message and resulting task switches

other interrupts, on our hardware the reschedule takes effect within roughly 10 microseconds after receiving the ICMP message.

\section{Future Work}

Our approach of instrumenting selected locations in the kernel is essential for performance issues on the OS level. Future versions of our tools may consider more process related data or additional event types. For a comprehensive understanding, one needs insight from the application point of view. For example, better support for MPI applications or multithreaded Java programs is desirable, e.g. in order to recognize threads of execution or process groups and observe effects of communication patterns between them. Ideally, one would like to combine our approach with one or more of the many application level oriented performance analysis tools. The process IDs that we record on the OS level offer the natural basis for an integration with log file data collected in user level space. Integration of the data either would be done by a separate tool or integrated into our visualization tool.

When combining log files from multiple hosts into a single display, we assume that all logs were started at the same time and the drift between the clocks is negligible. Otherwise, the display may show a horizontal offset error on the time line. Our modifications to the kernel allow for broadcasting special ICMP messages that remotely start/stop the logging mechanism, using the same infrastructure as for the remote schedule feature. First experience shows that this way, the skew is not worse than 10 microseconds even via a slow $10 \mathrm{MBit} / \mathrm{s}$ Ethernet connection. However, intercepting hardware interrupts may contribute to further delay. To eliminate this source of error, we may follow recent work on network time synchronization[2]. This work shows that with broadcasts, the difference in the arrival time is typically small and OS related skew can be minimized by recording the system clock's current time immediately at interrupt 
time upon receiption of a packet. Consequently, we would try to record the system time clock upon receipt of an ICMP message as early as possibly and use it as reference start time for the logging process.

\section{Related Work}

Almost all software performance analysis tools that we know of focus on either application or communication library level. The classical UNIX tool gprof [7] and the Java tool hprof[8] follow the stochastical profiling approach by interrupting program execution in regular intervals and recording program status information such as the current execution stack.

$\mathrm{DiP}[9]$ is a collection of tools for recording a stream of events at PVM or MPI level by instrumenting the underlying communication library. DiP's visualization tool PARAVER uses time lines for visualization and supports textual and graphical display of user defined functions on lists of events.

SCALEA[14] supports automatic and manual instrumentation of user level source code, using a novel representation of code regions in the course of call graph analysis that also covers loops, I/O and communication statements.

Recent versions of Paradyn[1] also follow the approach of examining call graphs. Paradyn uses dynamic instrumentation to insert and delete measurement instrumentation as a program runs. Visualization under Paradyn is mostly based on statistical diagrams and search history graphs rather than time lines.

Due to a growing number of performance analysis tools, some work focuses on standardization. MPI[5] provides a built-in interface for profiling tools. Similar work has been suggested for OpenMP[11]. JavaPSL[3] is a generic language for describing experiment-related data and performance properties, serving as a substitute for specific log file formats and including data of the experiment's environment. Gerndt et al.[6] present an aproach with automatic performance problem search based on the performance properties specification language ASL.

Tamches and Miller[13] present a framework for dynamically instrumenting the Solaris kernel by performing control flow analysis on the kernel machine code and patching it at runtime.

Feng et al.[4] present a system that conceptionally comes pretty close to our approach. Still, their implementation differs in some important details such as the use of SMP locks which our design avoids.

\section{Conclusion}

In this paper, we presented a set of tools for performance analysis that collects OS level data by instrumenting selected code in the OS kernel. We showed that the logging overhead can be kept small and taken into account in the analysis. Particular challenges and solutions on this approach inlude peculiarities of SMP machines. Using our Java-based visualization tool, we could gain first experience with our tools upon verifying the effect of a remote schedule feature that we 
implented in the kernel. We discussed how our approach may be combined with user level space performing analysis and how the synchronization of time lines across multiple machines may be further improved. The results of our work are publicly available at http://www.ipd.uka.de/ reuter/tools/.

\section{References}

1. Harold W. Cain, Barton P. Miller, and Brian J.N. Wylie. A callgraph-based search strategy for automated performance diagnosis. In Proc. of Euro-Par 2000 Parallel Processing, pages 108-122, 2000.

2. Jeremy Elson, Lewis Girod, and Deborah Estrin. Fine-grained network time synchronization using reference broadcasts. In Proc. of the Fifth Symposium on Operating Systems Design and Implementation (OSDI 2002), Boston, MA., December 2002.

3. Thomas Fahringer and Clóvis Seragiotto Júnior. Modeling and detecting performance problems for distributed and parallel programs with JavaPSL. In Proceedings of the 2001 ACM/IEEE conference on Supercomputing, November 2001.

4. W. Feng, M. Gardner, and J. Hay. The magnet toolkit: Design, evaluation, and implementation. Journal of Supercomputing, 23(1):67-79, August 2002.

5. Message Passing Interface Forum. MPI: A message-passing interface standard, June 1995. http://www.mpi-forum.org/docs/mpi-11.ps.

6. M. Gerndt, A. Schmidt, M. Schulz, and R. Wismüller. Performance analysis for teraflop computers - a distributed automatic approach. In Euromicro Workshop on Parallel, Distributed, and Network-based Processing, pages 23-30, Gran Canaria, January 2002.

7. Susan L. Graham, Peter B. Kessler, and Marshall K. McKusick. Gprof: A call graph execution profiler. In Proceedings of the SIGPLAN '82 symposium on Compiler construction, pages 120-126, Boston, Massachusetts, United States, June 1982.

8. Stuart Halloway. Using HPROF to tune performance, January 2000. http://developer.java.sun.com/developer/TechTips/2000/tt0124.html\#tip2.

9. Jesús Labarta, Sergi Girona, Vincent Pillet, Toni Cortes, and Luis Gregoris. DiP: A parallel program development environment. In Proc. of the 2nd International Euro-Par Conference, 1996.

10. Oak Ridge National Laboratory. PVM: Parallel virtual machine. http://www.epm. ornl.gov/pvm/pvm_home.html.

11. Bernd Mohr, Allen D. Malony, Sameer Shende, and Felix Wolf. Towards a performance tool interface for OpenMP: An approach based on directive rewriting. In Proc. of Third European Workshop on OpenMPI (EWOMP 2001), Barcelona, September 2001.

12. John Postel. Request for comments RFC 792: Internet control message protocol, September 1981. http://www.ietf.org/rfc/rfc0792.txt.

13. Ariel Tamches and Barton P. Miller. Fine-grained dynamic instrumentation of commodity operating system kernels. In Proc. of Operating Systems Design and Implementation (OSDI 1999), 1999.

14. Hong-Linh Truong and Thomas Fahringer. SCALEA: A performance analysis tool for distributed and parallel programs. In Proc. of the 8th International Euro-Par Conference, LNCS 2400, Paderborn, Germany, August 2002. 